\title{
High-resolution x-ray tomographic morphological characterisation of electrospun nanofibrous bundles for tendon and ligament regeneration and replacement
}

\author{
Alberto Sensini ${ }^{1}$, Luca Cristofolini $^{1,2}$, Maria Letizia Focarete ${ }^{2,3}$, Juri Belcari ${ }^{1}$, Andrea \\ Zucchelli ${ }^{1}$, Alexander $\mathrm{Kao}^{4}$, Gianluca Tozzi ${ }^{4}$ \\ ${ }^{1}$ Department of Industrial Engineering, Alma Mater Studiorum - Università di \\ Bologna, 40131 Bologna, Italy \\ ${ }^{2}$ Health Sciences and Technologies - Interdepartmental Center for Industrial Research \\ (HST-ICIR), Alma Mater Studiorum - Università di Bologna, 40064 Ozzano \\ dell'Emilia, Bologna, Italy \\ ${ }^{3}$ Department of Chemistry "G. Ciamician" and National Consortium of Materials \\ Science and Technology (INSTM, Bologna RU), Alma Mater Studiorum - Università \\ di Bologna, 40126 Bologna, Italy \\ ${ }^{4}$ Zeiss Global Centre, School of Engineering, University of Portsmouth, Portsmouth \\ PO1 3DJ, United Kingdom
}

\section{Corresponding author:}

Luca Cristofolini

Department of Industrial Engineering

School of Engineering and Architecture

University of Bologna

Viale Risorgimento, 2

40136 Bologna, Italy

e-mail: luca.cristofolini@unibo.it 


\section{Summary}

Repair of ligaments and tendons requires scaffolds mimicking the spatial organization of collagen in the natural tissue. Electrospinning is a promising technique to produce nanofibers of both resorbable and biostable polymers with desired structural and morphological features. The aim of this study was to perform high-resolution x-ray tomography (XCT) scans of bundles of Nylon6.6, pure PLLA and PLLA-Collagen blends, where the nanofibers were meant to have a predominant direction. Characterisation was carried out via a dedicated methodology to firmly hold the specimen during the scan and a workflow to quantify the directionality of the nanofibers in the bundle. XCT scans with 0.4 and 1.0 micrometer voxel size were successfully collected for all bundle compositions. Better image quality was achieved for those bundles formed by thicker nanofibers (i.e. 0.59 micrometers for pure PLLA), whereas partial volume effect was more pronounced for thinner nanofibers (i.e. 0.26 micrometers for Nylon6.6). As expected, the nanofibers had a predominant orientation along the axis of the bundles (more than $20 \%$ of the nanofibers within $3^{\circ}$ and more than $60 \%$ within $18^{\circ}$ from the bundle axis), with a Gaussian-like dispersion in the other directions. The directionality assessment was validated by comparison against a similar analysis performed on SEM images: the XCT analysis overestimated the amount of nanofibers very close to the bundle axis, especially for the materials with thinnest nanofibers, but adequately identified the amount of nanofibers within $12^{\circ}$.

\section{Key words:}

Electrospinning; X-ray tomography; Tissue engineering; Tendon and Ligament repair and replacement; Nanofibers. 


\section{Introduction}

Injuries of tendons and ligaments are still an unsolved clinical problem, and surgical treatments are far from satisfactory (Santos, Rodrigues, Domingues, Reis \& Gomes, 2017). Tendon and ligaments such as the rotator cuff, the anterior cruciate ligament and the Achilles tendon are among the most commonly injured tissues in relation to both sporting activities and degenerative process associated with chronic inflammation (Harvie et al., 2004; Rees, Wilson \& Wolman, 2006; September, Schwellnus, Collins \& Gibson, 2007; Woo, Abramowitch, Kilger \& Liang, 2006). The repair of these tissues is particularly difficult since their complex hierarchical structure, composed of predominantly aligned collagen fibers at different levels of aggregation (Goh, Listrat \& Béchet, 2014; Kannus, 2000; Kastelic, Galeski \& Baer, 1978), is difficult to replicate. Another critical aspect is given by the difficulty in restoring the natural mechanical properties, which are intrinsically non-linear (Goh et al., 2014; Santos et al., 2017) after an injury. Among all the strategies to manage these kind of injuries tissue engineering is very promising, as it allows regeneration of the native tissue (Santos et al., 2017). Thanks to its capability to produce nanofibers from polymeric solutions of both resorbable and biostable materials, electrospinning, is one of the most promising technologies in the field of regenerative medicine (Uquillas, Pacelli, Kobayashi \& Uquillas, 2017). In fact, electrospinning technology allows to fabricate scaffolds that reproduce the structure of collagen fibrils (Verdiyeva, Koshy, Glibbery, Mann \& Seifalian, 2015). In order to increase the mechanical properties of the nanofibrous mats, 3D scaffolds of both aligned and twisted nanofibers (called respectively bundles and yarns) were proposed (O'Connor \& McGuinness, 2016). In particular the multiscale morphology of the bundles is also suitable to mimic the multiscale morphology of tendon and ligament fascicles (O’Connor \& McGuinness, 2016). 
Generally, the morphological investigation of electrospun mats, bundles and yarns is performed using scanning electron microscopy (SEM) (O’Connor \& McGuinness, 2016; Verdiyeva et al., 2015). This characterization technique, however, only produces information related to the morphology of the surface, and does not allow examining the internal volume of the scaffolds. To overcome this limitation, $x$-ray computed tomography was employed to study electrospun polymeric fibers. Micro-computed tomography (micro-CT) is available from the late Eighties with a resolution down to few micrometers (Dover, Elliott, Boakes \& Bowen, 1989; Flannery et al., 1987). More recently, high-resolution x-ray tomography systems with a sub-micrometer resolution have become available, and are referred to as XCT in this paper. However, XCT imaging of electrospun fibers poses a number of technical issues: (i) the low attenuation of the polymeric fibers; (ii) the difficulty in avoiding micro-movements of the specimens (which are highly deformable) during imaging, which would produce artifacts; and (iii) the difficulty to conjugate nanometric dimension of the fibers and high-resolution in terms of voxel size of the scans. For these reasons, only few studies employed highresolution XCT imaging of electrospun nanofibers, mostly referring to random nano/microfibrous electrospun mats. (Farrugia et al., 2013) produced tomographic images with a voxel size of 0.79 micrometers of a poly( $\varepsilon$-caprolactone) electrospun scaffold made of random microfibers with a mean diameter of 7 micrometers, estimating the porosity of the scaffolds. The composition of the scaffolds and the micrometric diameter of the fibers allowed to clearly distinguish the fibers' contours. (Kogikoski et al., 2017) used tomographic images with a voxel size of 3.37 micrometers to visualize mats of random nanofibers (mean diameters $0.6-0.3$ micrometers) of $\operatorname{poly}(\varepsilon)$ caprolactone (PCL) with polyaniline (PANI) doped with the amino acid N-acetyl-Lcysteine (NAC) blends. Also in that work the porosity was investigated. However, as 
the voxel size exceeded the fibers diameter visualization of the individual fibers was not possible, but just an overview of the density of the scaffolds was showed. (Bradley, Robinson \& Yusuf, 2017) acquired tomographic images with sub-micrometer resolution ( 0.13 micrometers of voxel size) to investigate the cells' infiltration on electrospun microfibrous (mean fiber diameters 4 micrometers) mats of poly(lactide-co-glycolide) (PLGA). Thanks to the segmentation and the post processing methods, but also to the voxel size being one order of magnitude lower than the diameters of the fibers, the fibers' contours and the cells were highly defined. However, due to the small voxel size selected, just a short portion of the samples was scanned.

Moving from mats of electrospun fibers to more complex configurations, such as bundles or yarns, the difficulties related to centring the specimen in the scanner x-ray microscope, and to micro-movements during the scans increase dramatically. Just two works were presented in the literature about tomographic scans on electrospun bundles and yarns for tendon and ligament regeneration. (Bosworth, Alam, Wong \& Downes, 2013; Bosworth, Rathbone, Bradley \& Cartmell, 2014) showed tomographic images, with a voxel size of 0.61 micrometers, of electrospun nanofibrous yarns of PCL (mean fiber diameters 0.4 micrometers) as-spun and after cell culture. Their tomograms allowed showing the bundles surface and the regenerated tissue on their surfaces. However the fibers' contours were not clearly distinguishable, due to a significant partial volume effect. Very recently, tomographic scans with a resolution of 0.33 micrometers were produced using synchrotron x-ray phase contrast imaging of mats of biodegradable polyester nanofibers with diameters between 1.9 and 3.7 micrometers (Maksimcuka et al., 2017).

One limitation of most of the works mentioned above is that the electrospun samples (either mats, or yarns or bundles) were placed inside a plastic tube for the scans to avoid 
micro-movements due to the flexibility of the scaffolds. The outer diameter of the tube forces to enlarge the volume of interest, thus reducing the resolution of the scan. Furthermore, the tube adds a shielding effect to the electrospun samples: even if the tube is made of a similar material, it adds significant absorption because its walls are much thicker, and because the tube material does not have significant porosity. This can result in a loss of image quality. Recently, (Sensini et al., 2017) investigated electrospun bundles of aligned nanofibers of PLLA and collagen blends (mean fiber diameters of 0.3 micrometers), including a XCT feasibility study with a voxel size of 0.4 micrometers. In that study, the individual fibers were well-defined, and no artefacts due to micro-movements were observed.

The aims of the present study were: 1) to acquire high-resolution x-ray tomography scans of electrospun nanofibrous bundles made of pure PLLA, Nylon6.6 and PLLA/Coll blends in different percentages; and 2) to define a post processing workflow on the XCT scans to evaluate the alignment of the nanofibers in all the bundles' volume. In order to validate the alignment based on XCT, a similar investigation was performed on scanning electron microscopy (SEM) images. 


\section{Materials and methods}

\section{Electrospun bundles preparation}

Polymeric solutions were obtained using the reported materials: acid soluble collagen type I (Coll) from bovine skin kindly provided by Kensey Nash Corporation d/b/a DSM Biomedical (Exton, USA); Poly-L-lactic acid (PLLA) (Lacea H.100-E, Mw = 8.4 × 104 $\mathrm{g} \mathrm{mol}^{-1}, \mathrm{PDI}=1.7$ ) was purchased from Mitsui Fine Chemicals (Dusseldorf, Germany); Nylon6.6 kindly provided by DuPont (Wilmington, USA); trifluoroacetic acid (TFA) was purchased by Carlo Erba (Milan, Italy), 2,2,2-trifluoroethanol (TFE), 1,1,1,3,3,3hexafluoro-2-propanol (HFIP), dichloromethane (DCM), dimethylformamide (DMF), acetone (AC), N-(3-Dimethylaminopropyl)-N'-ethylcarbodiimide hydrochloride (EDC) and N-Hydroxysuccinimide (NHS) were purchased by Sigma-Aldrich (Saint Louis, USA) and used as received. The PLLA samples were produced from PLLA dissolved in a solution of DCM and DMF. The PLLA/Coll-75/25 and PLLA/Coll-50/50 blends were dissolved in a solution of TFE and HFIP. The Nylon6.6 was dissolved in a solution of TFA and AC. For more details, the reader is referred to our previous work (Sensini et al., 2017).

A laboratory electrospinning machine (Spinbow Lab Unit, Spinbow S.r.1., Bologna, Italy), equipped with a linear sliding spinneret (two syringes for PLLA and PLLA/Coll blends; four syringes for Nylon6.6) and a high-speed rotating drum collector (diameter $=150 \mathrm{~mm}$; length $=500 \mathrm{~mm}$; rotational speed $=2900 \mathrm{rpm}$ ) was used to produce mats of aligned nanofibers as shown in Figure 1(A). To electrospin the solutions a syringe pump (KD Scientific 200 series, Holliston, USA) and glass syringes connected to stainless steel blunt-ended needles with Teflon tubes were used. Electrospinning was performed at room temperature and relative humidity $20-30 \%$. The pure PLLA, PLLA/Coll-75/25 
and PLLA/Coll-50/50 blends were electrospun with the parameters previously reported (Sensini et al., 2017). Nylon6.6 was electrospun with $20 \mathrm{kV}$ voltage, $0.5 \mathrm{~mL} / \mathrm{hour}$ flow rate per syringe and $160 \mathrm{~mm}$ needle-collector distance. After the spinning sessions, the mats of electrospun aligned nanofibers were cut in strips and manually wrapped to obtain bundles with mean diameters of 550-650 micrometers as shown in Figure 1(B) - (C) (D). This diameter is in the same range as tendon and ligament fascicles (Goh et al., 2014; Kannus, 2000; Kastelic et al., 1978). The bundles of PLLA/Coll-75/25 and PLLA/Coll-50/50 were crosslinked by immersion for 24 hours at room temperature in a crosslinking solution of EDC and NHS $0.02 \mathrm{M}$ in ethanol and distilled water (adapted from (Kim, Song \& Kim, 2005)).

\section{XCT Imaging}

The three-dimensional high-resolution scans were acquired using a XCT (Versa 510, ZEISS, Pleasanton, CA, USA), with two different isotropic voxel sizes for the reconstructed images. XCT scans of one specimen for each composition (Nylon6.6, pure PLLA, PLLA/Coll-75/25 and PLLA/Coll-50/50 as-spun and immediately after crosslinking) were acquired. In order to eliminate artefacts caused by micromovements, custom made rectangular masks $(31 \times 8 \times 0.5 \mathrm{~mm})$ made of polyethylene terephthalate $(\mathrm{PET})$, with a central rectangular window $(13 \times 5 \mathrm{~mm})$, were used to stabilize the bundles in the XCT chamber. Specimens of $36 \mathrm{~mm}$ in length were mounted vertically in the centre of the windows, with the extremities fixed on the masks with bicomponent glue as shown in Figure 2(AI). This solution was adapted from a first prototype previously applied in a feasibility study of XCT on PLLA/Coll bundles (Sensini et al., 2017). 
Settings used for each voxel size were as follows:

(i) Voxel size of 1.0 micrometer: $40 \mathrm{kV}$ Voltage, $3 \mathrm{~W}$ Power, 75.5 microAmpere tube current, $8 \mathrm{sec}$ exposure time, images collected at rotational steps of $0.18^{\circ}$ over $360^{\circ}$, for a scanning time of approximately 6 hours;

(ii) Voxel size of 0.4 micrometers: $40 \mathrm{kV}$ Voltage, $3 \mathrm{~W}$ Power, 75.5 microAmpere tube current, $14 \mathrm{sec}$ exposure time, images collected at rotational steps of $0.18^{\circ}$ over $360^{\circ}$, for a scanning time of approximately 10 hours.

Post processing: analysis of directionality from XCT images

The images were reconstructed using the Scout-and-Scan Reconstructor software (ZEISS), and were visualised using XM3DViewer1.2.8 software (ZEISS). Before the Directionality analysis, the XCT stack of cross-sectional slices of each bundle were axially resliced using the command Reslice of ImageJ Figure 2(AII). In order to quantify the directionality of the nanofibers, the scans at 0.4 micrometers of voxel size of all compositions were analysed with ImageJ (Liu, 1991), using a dedicated plugin called Directionality (Schindelin et al., 2012; Schneider, Rasband \& Eliceiri, 2012). The Directionality histogram reported the amount of nanofibers as a function of the fiber orientation using a Local Gradients orientation method applied to each slice. The mean and standard deviation between slices was then computed.

\section{SEM Imaging}

Scanning electron microscopy (SEM) observations were carried out using a Phenom ProX (Eindhoven, Netherlands) at an accelerating voltage of $15 \mathrm{kV}$, on samples sputtercoated with gold Figure 2(BI). For each of the bundle compositions (i.e. Nylon6.6, pure 
PLLA, PLLA/Coll-75/25 and PLLA/Coll-50/50 both as-spun and crosslinked) ten images were acquired with a magnification of $8000 \mathrm{x}$. The diameter of the nanofibers was measured on the SEM images using the software ImageJ (Liu, 1991). The distribution of diameters (mean and standard deviation) was measured for about 200 nanofibers, for each bundle composition.

\section{Validation of XCT imaging based on SEM images}

In order to validate the Directionality investigation on the XCT scans of the bundles, the same directionality investigation was performed as a comparison, on ten SEM images for each of the compositions, as shown in Figure 2(BII).

\section{Results}

\section{Bundles nanofibers morphology from SEM and XCT imaging}

All the bundles in the different compositions had a mean diameter in the range of 550650 micrometers as measured in the SEM images. Furthermore, the SEM investigation revealed that the nanofibers for each composition and each treatment condition had a well-defined morphology with no defects such as beads, as shown in Figure 3. The nanofibers had comparable diameters (mean and standard deviation) for all compositions and all treatments: Nylon6.6 $0.26 \pm 0.04$ micrometers; pure PLLA 0.59 \pm 0.14 micrometers; PLLA/Coll-75/25 as-spun $0.33 \pm 0.08$ micrometers; PLLA/Coll75/25 crosslinked $0.30 \pm 0.06$ micrometers; PLLA/Coll-50/50 as-spun $0.39 \pm 0.13$ micrometers; PLLA/Coll-50/50 crosslinked 0.40 \pm 0.08 micrometers.

The specimen mounting setup for the XCT scans successfully prevented the artefacts of micro-movements, while permitting to centre the bundle in the scanning window of the 
XCT as shown in Figure 2(AI). After the three-dimensional reconstruction at both voxel sizes, sharp high-resolution images were successfully obtained, as shown in Figure 4 and Figure 5. The reconstructions with 1.0 micrometer voxel size provided an overview of the each specimen. At 0.4 micrometer voxel size, the nanofibers were clearly distinguishable. Such high-resolution images allowed zooming-in a section of the specimens, both on its surface and inside the volume of the bundles.

The XCT images highlighted some loss of material inside the bundles of the two PLLA/Coll blends, due to the crosslinking process, as shown in Figure 5.

\section{Directionality of the nanofibers from XCT images}

For all the bundles, the Directionality analysis on the XCT stacks, confirmed a preferential alignment of the nanofibers, which were predominantly close to the axis of the bundle, and with a progressive Gaussian-like dispersion (Figure 6, 7 and 8). The single-polymer bundles presented lower peaks in the range of $0^{\circ}-3^{\circ}$ (Nylon6.6 $29.5 \pm 2.4 \%$ and PLLA $21.5 \pm 1.8 \%$ ) compared to the PLLA/Coll blends, both as-spun (PLLA/Coll-75/25 as-spun 38.4 $\pm 4.3 \%$ and PLLA/Coll-50/50 as-spun 39.4 $\pm 3.5 \%$ ) and crosslinked (PLLA/Coll-75/25 crosslinked $31.4 \pm 2.5 \%$ and PLLA/Coll-50/50 crosslinked $35.3 \pm 2.7 \%$ ). A small fraction of nanofibers were oriented in the range of $87^{\circ}-90^{\circ}$ for the single-polymer bundles (Nylon6.6 $0.4 \pm 0.1 \%$ and PLLA $0.6 \pm 0.1 \%$ ), the PLLA/Coll blends, both as-spun (PLLA/Coll-75/25 as-spun $0.5 \pm 0.2 \%$ and PLLA/Coll50/50 as-spun $0.4 \pm 0.1 \%$ ) and crosslinked (PLLA/Coll-75/25 crosslinked $0.5 \pm 0.1 \%$ and PLLA/Coll-50/50 crosslinked 0.4 $\pm 0.1 \%$ ). 
The comparison between the XCT and SEM images focused on the analysis of Directionality. The analysis of the XCT stacks resulted in a higher estimation of the axial alignment of the nanofibers: for all the compositions, the amount of nanofibers in the $0^{\circ}-3^{\circ}$ range was systematically higher when estimated from the XCT scans than from the SEM images (Figure 6, 7 and 8). In the range from $3^{\circ}$ to $21^{\circ}$ (for Nylon6.6) or $24^{\circ}$ for the other compositions the XCT underestimated the amount of nanofibers, compared to the SEM images (the discrepancy was smaller compared to the $0^{\circ}-3^{\circ}$ range). The discrepancy between the amount of nanofibers between $21^{\circ}$ and $90^{\circ}$ estimated from the XCT scans and SEM images was well below 1\%, for all the compositions.

The largest amount of nanofibers in the $0^{\circ}-3^{\circ}$ range were measured in the XCT scans of the PLLA/Coll blends. The differences between the XCT and SEM amount histograms in the $0^{\circ}-3^{\circ}$ range were relatively small for the Nylon6.6 (3.2\% discrepancy) and the PLLA (0.8\%). The differences between the XCT and SEM were larger for the blends containing collagen (15.6\% for the PLLA/Coll-75/25 as-spun, $12.6 \%$ for the PLLA/Coll-75/25 crosslinked and $13.6 \%$ for the PLLA/Coll-50/50 as-spun, $14.9 \%$ for the PLLA/Coll-50/50 crosslinked).

The discrepancy between the amount of nanofibers estimated based on the XCT scans and SEM images was smaller if a larger range of angles was considered $\left(0^{\circ}-12^{\circ}\right)$ : the difference for the PLLA/Coll blends did not exceed 2\%; the largest difference was found for the Nylon6.6 (10\% difference) and pure PLLA (6\% difference). 


\section{Discussion}

The first aim of the present study was to obtain XCT scans of electrospun bundles of different materials in order to compare their morphology with the multiscale structure of tendon and ligament fascicles and fibrils (Goh et al., 2014; Kannus, 2000; Kastelic et al., 1978). For this reason, we developed a setup to avoid micro-movements during the XCT scans, and we defined a post processing workflow on the XCT scans to evaluate the alignment of the nanofibers in all the bundles' volume. To validate the alignment measured from the XCT, a comparison was performed against a similar investigation with SEM images (SEM).

We produced bundles of nanofibers of biostable and resorbable materials, morphologically and hierarchically similar, in terms of diameters and fiber alignment to the fascicles and fibrils of the human tendons and ligaments (Fang \& Lake, 2016; Goh et al., 2014; Kannus, 2000; Kastelic et al., 1978; Radaelli et al., 2017). The surface morphology and alignment of the nanofibers were assessed through SEM investigation (Figure 3). In order to analyse the 3D-morphology of the bundles and verify the alignment of the nanofibers even in their internal part, an XCT characterization with two different voxel sizes (1.0 and 0.4 micrometers) was performed on all the different compositions (Figure 4 and 5). For all the compositions, sharp XCT scans were obtained, for both voxel sizes (Figures 4 and 5). The two different voxel sizes were chosen to have an overview of the bundles (1.0 micrometer) and a zoom-in on the fibers (0.4 micrometers). Because of the nanofibers diameters $(550-250 \mathrm{~nm})$, increasing the resolution of the scan resulted in a better detection of the fibers contours (Figure 4 and 5), basically because a 0.4 micrometer voxel is more likely contains just one nanofiber. In fact, when increasing the dimension of the voxel more than one fiber could be 
contained in the same voxel, thus making it impossible to identify the nanofibers individually.

No images were affected by artefacts caused by micro-movements, and were sufficiently sharp to allow identification of the individual nanofibers. The main element to prevent micro-movements of such slender specimens was the fixture developed to hold the bundle during XCT imaging (Figure 2). This dedicated setup allowed placing the X-ray source and detector of the XCT as close as few millimetres to the specimen surface. Furthermore, the design of the support fixture did not enclose the specimen into any additional material, which could compromise the scan quality. Other works on electrospun materials used polyamide tubing (Bosworth et al., 2014) or Kapton tubing (Bradley et al., 2017) as a support for the specimen during XCT imaging. With this configuration it is difficult to obtain a correct contrast between the walls of the tubing and the bundles, because of the low absorption of the electrospun polymers, as well as additional attenuation due to the tube itself.

The Directionality analysis on the XCT scans, confirmed a preferential axial alignment in the range of $0^{\circ}-3^{\circ}$ for all the bundles, and a progressive dispersion of the nanofibers in the range of $3^{\circ}-90^{\circ}$. This is similar to the physiological dispersion of collagen fibrils in the tendons and ligaments (Franchi, Trirè, Quaranta, Orsini \& Ottani, 2007; Goh et al., 2014; Radaelli et al., 2017). The fact that most (but not 100\%) of the fibers are aligned with the bundle axis is important to mimic the arrangement of the collagen in the tendons and ligaments. In fact, this structure of the bundles is expected to be biomimetic, to promote tissue regeneration, and to provide a non-linear mechanical response similar to that of the natural tissue (Goh et al., 2014). A related study has shown that three-dimensional multiscale morphology of scaffolds similar to those in the current study, as well as the particular use of PLLA/Coll blends permitted the bundles 
to better reproduce the biomechanical properties of human tendon and ligament fascicles (Goh et al., 2014; Sensini et al., 2017).

The comparison between the Directionality analyses performed on the XCT scans and SEM images showed generally a good agreement. It must be noted that the SEM inspection was assumed as a ground truth: however, SEM inspection only addresses the specimen's surface, whereas it cannot access the inner volume. The Directionality tests were performed on all the XCT stacks for each specimen (about 900 slices), while for the SEM the analysis relied on 10 images from the surface of each bundle. This can partially explain the difference between XCT and SEM assessment of directionality. The largest discrepancy between the two examination methods was found in the estimation of the amount of nanofibers aligned within $3^{\circ}$ from the axis of the bundle, where the XCT scan provided a considerable overestimation. While this discrepancy was within $3 \%$ for the bundles of Nylon6.6 and the PLLA, larger discrepancies were observed for the PLLA/Coll blends (between $12.6 \%$ and 15.6\%). However, if a larger range of directionality was considered (i.e. $0^{\circ}-12^{\circ}$ ) better agreement was found between directionality assessed form XCT scans and SEM images (2\% discrepancy for the PLLA/Coll blends, $6 \%$ for pure PLLA and 10\% for Nylon6.6). This seems to indicate that XCT scans are less suited to assess the amount of nanofibers in a very narrow range of angles, whereas they can reliably quantify the amount of nanofibers in a range of $12^{\circ}$. The reason of this behaviour is probably the partial volume effect. The nanofibers of pure PLLA had a mean diameter ( 0.59 micrometers) larger than the voxel size $(0.4$ micrometers). Thus, most of the nanofibers were clearly distinguishable, and consequently their direction was more accurately captured. Conversely, PLLA nanofibers thinner than the voxel size were not clearly recognised in the XCT scan and therefore they contributed to overestimate the amount in the range of $0^{\circ}-21^{\circ}$. The partial 
volume effect was more pronounced for the XCT scans of the Nylon6.6 bundles, where the mean diameter of the nanofibers ( 0.26 micrometers) was smaller than the voxel size. This effect was further increased for the PLLA/Coll blends as the collagen: in fact it is well well-know that it is difficult to obtain tomographic images of the collagen due to its low absorption of x-ray radiation (Balint, Lowe \& Shearer, 2016; Zidek et al., 2016).

In our study on electrospun bundles, the XCT scans had a greyscale similar to the background, because of the space between nanofibers, and because the nanofibers dimensions. This made the detection of the nanofibers direction even more difficult. To obtain tomographic scans with a resolution of 0.33 micrometers, synchrotron X-ray phase contrast was used (Maksimcuka et al., 2017): while this allowed identifying the individual polyester nanofibers (diameter between 1.9 and 3.7 micrometers), it also caused significant material modification due to the high radiation dose. Some works used contrast agents to enhance radiopacity during $\mathrm{CT}$ scans of portions of anterior cruciate ligaments and patellar tendons where the effects of phosphotungstig acid (PTA) and iodine solution (IKI) staining were compared, in order to increase the visualization of the tissues, down to the fascicle level (Shearer et al., 2014; Shearer, Bradley, HidalgoBastida, Sherratt \& Cartmell, 2016). Unstained rat common carotid artery embedded in paraffin, was successfully XCT scanned using a voxel size of 0.5 micrometers, showing collagen fibrils (Walton et al., 2015). 


\section{Conclusions}

We have shown the feasibility of assessing the morphology of electrospun polymeric bundles by means of high-resolution computed tomography (XCT). We were able to quantify the directionality of the nanofibers in bundles with different biostable and bioresorbable compositions that produced a 3D multiscale scaffold able to replicate the morphology of the human tendon and ligament fascicles.

\section{Acknowledgments}

FP7 COST Action MP1206 'Electrospun Nano-fibers for bio inspired composite materials and innovative industrial applications' and the Italian Ministry of University and Research (MIUR) are acknowledged. Type I collagen was kindly provided by Kensey Nash Corporation d/b/a DSM Biomedical (Exton, USA). Michele Segala, Chiara Dazzi, Elisa Bendanti and Rachna Parwani are acknowledged for their help in crosslinking treatments, mechanical characterization and technical support in the XCT image acquisition respectively. The ZEISS Global Centre at the School of Engineering (University of Portsmouth) is acknowledged for providing XCT facilities. The mobility of Alberto Sensini was funded by university of Bologna (Marco Polo grant). 


\section{REFERENCES:}

Balint, R., Lowe, T. \& Shearer, T. (2016). Optimal contrast agent staining of ligaments and tendons for X-ray computed tomography. PLoS ONE, 11(4). https://doi.org/10.1371/journal.pone.0153552

Bosworth, L. A., Alam, N., Wong, J. K. \& Downes, S. (2013). Investigation of 2D and 3D electrospun scaffolds intended for tendon repair. Journal of Materials Science: Materials in Medicine, 24(6), 1605-1614. https://doi.org/10.1007/s10856-013-4911-8

Bosworth, L. A., Rathbone, S. R., Bradley, R. S. \& Cartmell, S. H. (2014). Dynamic loading of electrospun yarns guides mesenchymal stem cells towards a tendon lineage. Journal of the Mechanical Behavior of Biomedical Materials, 39, 175183. https://doi.org/10.1016/j.jmbbm.2014.07.009

Bradley, R. S., Robinson, I. K. \& Yusuf, M. (2017). 3D X-Ray Nanotomography of Cells Grown on Electrospun Scaffolds. Macromolecular Bioscience, 17(2), 1-8. https://doi.org/10.1002/mabi.201600236

Dover, S. D., Elliott, J. C., Boakes, R. \& Bowen, D. K. (1989). Three-dimensional Xray microscopy with accurate registration of tomographic sections. Journal of Microscopy, 153(2), 187-191. https://doi.org/10.1111/j.13652818.1989.tb00559.x

Fang, F. \& Lake, S. P. (2016). Modelling approaches for evaluating multiscale tendon mechanics. Interface Focus, 6(1), 20150044. https://doi.org/10.1098/rsfs.2015.0044

Farrugia, B. L., Brown, T. D., Upton, Z., Hutmacher, D. W., Dalton, P. D. \& Dargaville, T. R. (2013). Dermal fibroblast infiltration of poly( $\varepsilon$-caprolactone) scaffolds fabricated by melt electrospinning in a direct writing mode. Biofabrication, 5(2), 25001. https://doi.org/10.1088/1758-5082/5/2/025001

Flannery, B. P., Deckman, H. W., Roberge, W. G., Amico, K. L. D., Flannery, B. P., Deckman, H. W., ... Amico, K. L. D. (1987). Three -Dimensional X-ray Microtomography. Science, 237(4821), 1439-1444. https://doi.org/10.1126/science.237.4821.1439

Franchi, M., Trirè, A., Quaranta, M., Orsini, E. \& Ottani, V. (2007). Collagen Structure of Tendon Relates to Function. The Scientific World JOURNAL, 7, 404420. https://doi.org/10.1100/tsw.2007.92

Goh, K. L., Listrat, A. \& Béchet, D. (2014). Hierarchical mechanics of connective tissues: Integrating insights from nano to macroscopic studies. Journal of Biomedical Nanotechnology, 10(10), 2464-2507. https://doi.org/10.1166/jbn.2014.1960

Harvie, P., Ostlere, S. J., Teh, J., McNally, E. G., Clipsham, K., Burston, B. J., ... Carr, A. J. (2004). Genetic influences in the aetiology of tears of the rotator cuff. Sibling risk of a full-thickness tear. The Journal of Bone and Joint Surgery. British Volume, 86-B(5), 696-700. https://doi.org/10.1302/0301620X.86B5.14747

Kannus, P. (2000). Structure of the tendon connective tissue. Scandinavian Journal of Medicine and Science in Sports, 10(6), 312-320. https://doi.org/10.1034/j.16000838.2000.010006312.x 
Kastelic, J., Galeski, A. \& Baer, E. (1978). The Multicomposite Structure of Tendon. Connective Tissue Research, 6(1), 11-23. https://doi.org/10.3109/03008207809152283

Kim, H. W., Song, J. H. \& Kim, H. E. (2005). Nanofiber generation of gelatinhydroxyapatite biomimetics for guided tissue regeneration. Advanced Functional Materials, 15(12), 1988-1994. https://doi.org/10.1002/adfm.200500116

Kogikoski, S., Liberato, M. S., Factori, I. M., da Silva, E. R., Oliveira, C. L. P., Ando, R. A. \& Alves, W. A. (2017). Polycaprolactone-Polyaniline Blend: Effects of the Addition of Cysteine on the Structural and Molecular Properties. The Journal of Physical Chemistry C, 121(1), 863-877. https://doi.org/10.1021/acs.jpcc.6b10011

Liu, Z. (1991). Scale space approach to directional analysis of images. Applied Optics, 30(11), 1369-1373.

Maksimcuka, J., Obata, A., Sampson, W. W., Blanc, R., Gao, C., Withers, P. J., ... Poologasundarampillai, G. (2017). X-ray Tomographic Imaging of Tensile Deformation Modes of Electrospun Biodegradable Polyester Fibers. Frontiers in Materials, 4(December), 1-11. https://doi.org/10.3389/fmats.2017.00043

O’Connor, R. A. \& McGuinness, G. B. (2016). Electrospun nanofibre bundles and yarns for tissue engineering applications: A review. Proceedings of the Institution of Mechanical Engineers, Part H: Journal of Engineering in Medicine, 230(11), 987-998. https://doi.org/10.1177/0954411916656664

Radaelli, F., D’Alfonso, L., Collini, M., Mingozzi, F., Marongiu, L., Granucci, F., ... Sironi, L. (2017). $\mu$ MAPPS: a novel phasor approach to second harmonic analysis for in vitro-in vivo investigation of collagen microstructure. Scientific Reports, 7(1), 17468. https://doi.org/10.1038/s41598-017-17726-y

Rees, J. D., Wilson, A. M. \& Wolman, R. L. (2006). Current concepts in the management of tendon disorders. Rheumatology, 45(5), 508-521. https://doi.org/10.1093/rheumatology/kel046

Santos, M. L., Rodrigues, M. T., Domingues, R. M. A., Reis, R. L. \& Gomes, M. E. (2017). Regenerative Strategies for the Treatment of Knee Joint Disabilities. In J. M. Oliveira \& R. L. Ruis (Eds.), Regenerative Strategies for the Treatment of Knee Joint Disabilities (Vol. 21, pp. 349-371). Springer International Publishing. https://doi.org/10.1007/978-3-319-44785-8

Schindelin, J., Arganda-Carreras, I., Frise, E., Kaynig, V., Longair, M., Pietzsch, T., ... Cardona, A. (2012). Fiji: an open-source platform for biological-image analysis. Nature Methods, 9(7), 676-82. https://doi.org/10.1038/nmeth.2019

Schneider, C. A., Rasband, W. S. \& Eliceiri, K. W. (2012). NIH Image to ImageJ: 25 years of image analysis. Nature Methods, 9(7), 671-675. https://doi.org/10.1038/nmeth.2089

Sensini, A., Gualandi, C., Cristofolini, L., Tozzi, G., Dicarlo, M., Teti, G., ... Focarete, M. L. (2017). Biofabrication of bundles of poly ( lactic acid ) -collagen blends mimicking the fascicles of the human Achille tendon. Biofabrication, 9(1), 1-13. Retrieved from https://doi.org/10.1088/1758-5090/aa6204

September, A. V, Schwellnus, M. P., Collins, M. \& Gibson, W. (2007). Tendon and ligament injuries: the genetic component. British Journal of Sports Medicine, 41(4), 241-246. https://doi.org/10.1136/bjsm.2006.033035 
Shearer, T., Bradley, R. S., Hidalgo-Bastida, L. A., Sherratt, M. J. \& Cartmell, S. H. (2016). Three-dimensional visualisation of soft biological structures by X-ray computed micro-tomography. Journal of Cell Science, 129(13), 2483-2492. https://doi.org/10.1242/jcs.179077

Shearer, T., Rawson, S., Castro, S. J., Ballint, R., Bradley, R. S., Lowe, T., ... Cartmell, S. H. (2014). X-ray computed tomography of the anterior cruciate ligament and patellar tendon. Muscles, Ligaments and Tendons Journal, 4(2), 238-244. https://doi.org/10.11138/PER/2014.3.4.142

Uquillas, J. A., Pacelli, S., Kobayashi, S. \& Uquillas, S. (2017). Musculoskeletal Tissue Engineering: Tendon, Ligament, and Skeletal Muscle Replacement and Repair. In A. Hasan (Ed.), Tissue Engineering for Artificial Organs:

RegenerativeMedicine, Smart Diagnostics and PersonalizedMedicine (First Edit, pp. 465-523). Wiley. https://doi.org/https://doi.org/10.1002/9783527689934.ch15

Verdiyeva, G., Koshy, K., Glibbery, N., Mann, H. \& Seifalian, A. M. (2015). Tendon reconstruction with tissue engineering approach - A review. Journal of Biomedical Nanotechnology, 11(9), 1495-1523. https://doi.org/10.1166/jbn.2015.2121

Walton, L. A., Bradley, R. S., Withers, P. J., Newton, V. L., Watson, R. E. B., Austin, C. \& Sherratt, M. J. (2015). Morphological characterisation of unstained and intact tissue micro-architecture by X-ray computed micro- and nano-tomography. Scientific Reports, 5(March), 1-14. https://doi.org/10.1038/srep10074

Woo, S. L. Y., Abramowitch, S. D., Kilger, R. \& Liang, R. (2006). Biomechanics of knee ligaments: Injury, healing, and repair. Journal of Biomechanics, 39(1), 1-20. https://doi.org/10.1016/j.jbiomech.2004.10.025

Zidek, J., Vojtova, L., Abdel-Mohsen, A. M., Chmelik, J., Zikmund, T., Brtnikova, J., ... Kaiser, J. (2016). Accurate micro-computed tomography imaging of pore spaces in collagen-based scaffold. Journal of Materials Science: Materials in Medicine, 27(6), 1-18. https://doi.org/10.1007/s 10856-016-5717-2 


\section{Figures}
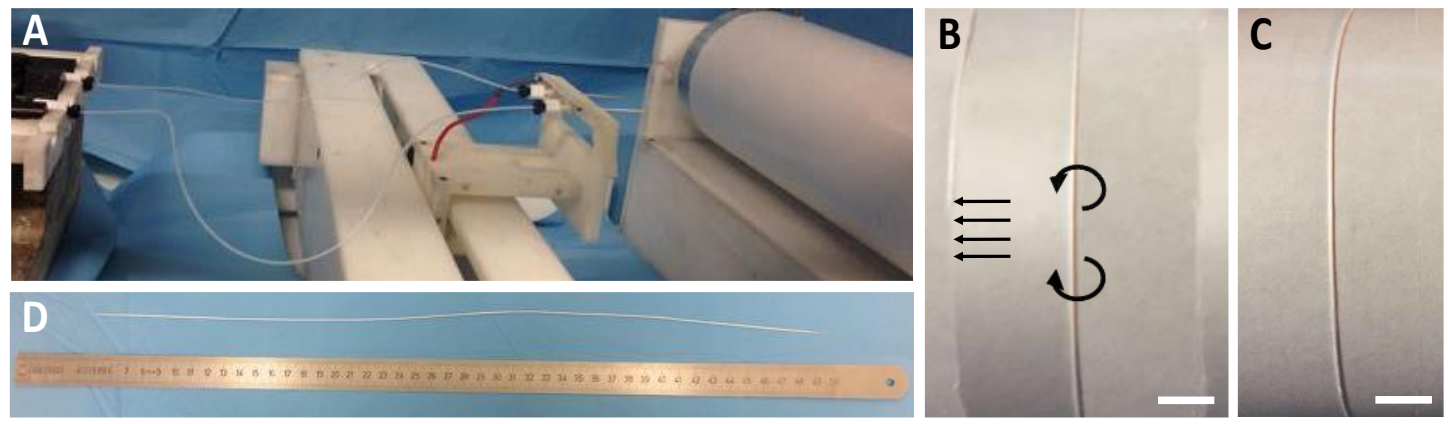

Fig. 1. (A) Electrospinning machine setup. (B) Image of an electrospun mat during the procedure of wrapping on the drum (scale bar $=20 \mathrm{~mm}$ ). (C) Image of a final bundle wrapped on the drum (scale bar $=20 \mathrm{~mm}$ ). (D) Overview of a bundle.

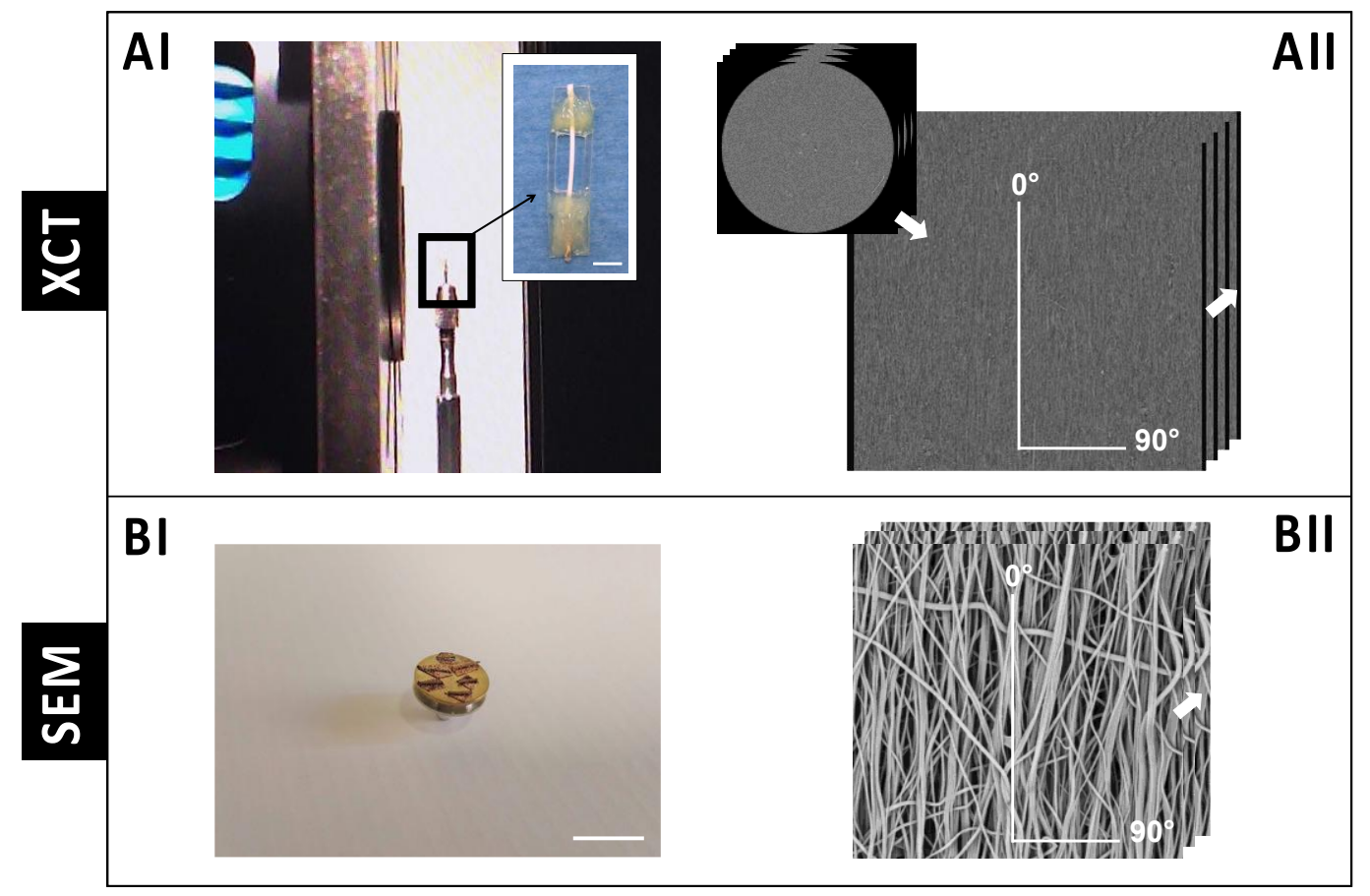

Fig. 2. Workflow of the Directionality analysis applied to the XCT and SEM images. (AI) Bundle mounted on the mask and ready for XCT scanning (scale bar $=10 \mathrm{~mm}$ ). (AII) Simplified representation of the Directionality procedure for the XCT images: the XCT stack consisting of cross-sections of the bundle at 0.4 micrometer voxel size was resliced in an axial stack by ImageJ. Then Directionality was applied to all the axial slices and angles were measured as indicated. (BI) Stub with five pieces of bundle, gold- 
sputtered and ready for SEM imaging (scale bar $=10 \mathrm{~mm}$ ). (BII) Simplified representation of the Directionality procedure for SEM images: 10 SEM images were acquired on different points of the surface of each bundle with a magnification of 8000x. The SEM images were investigated with Directionality with the same parameters of the XCT stacks.

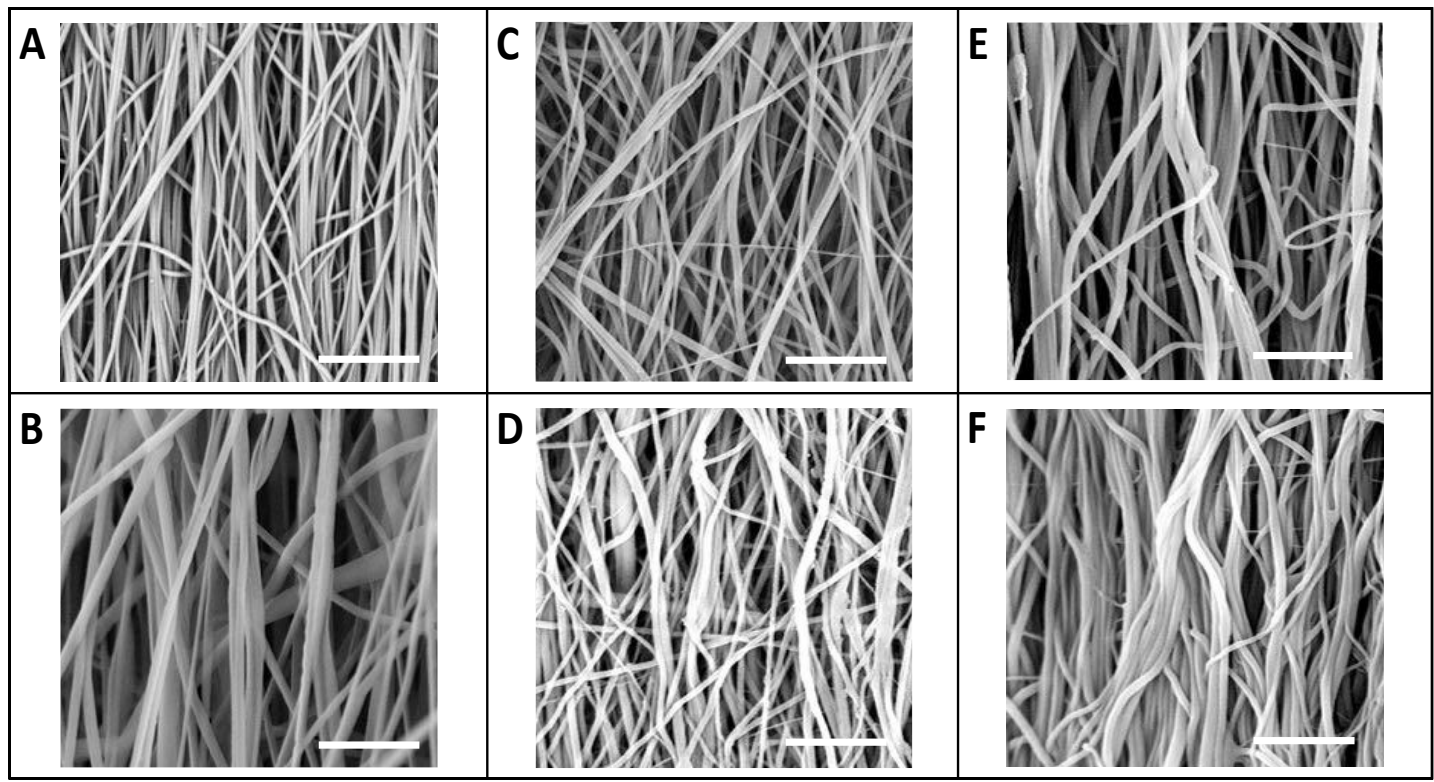

Fig. 3. SEM images of the nanofiber distribution within the bundles (magnification = 8000x, scale bar $=5$ micrometers). (A) Nylon 6.6. (B) PLLA. (C) PLLA/Coll-75/25 asspun. (D) PLLA/Coll-75/25 crosslinked. (E) PLLA/Coll-50/50 as-spun. (F) PLLA/Coll50/50 crosslinked. 


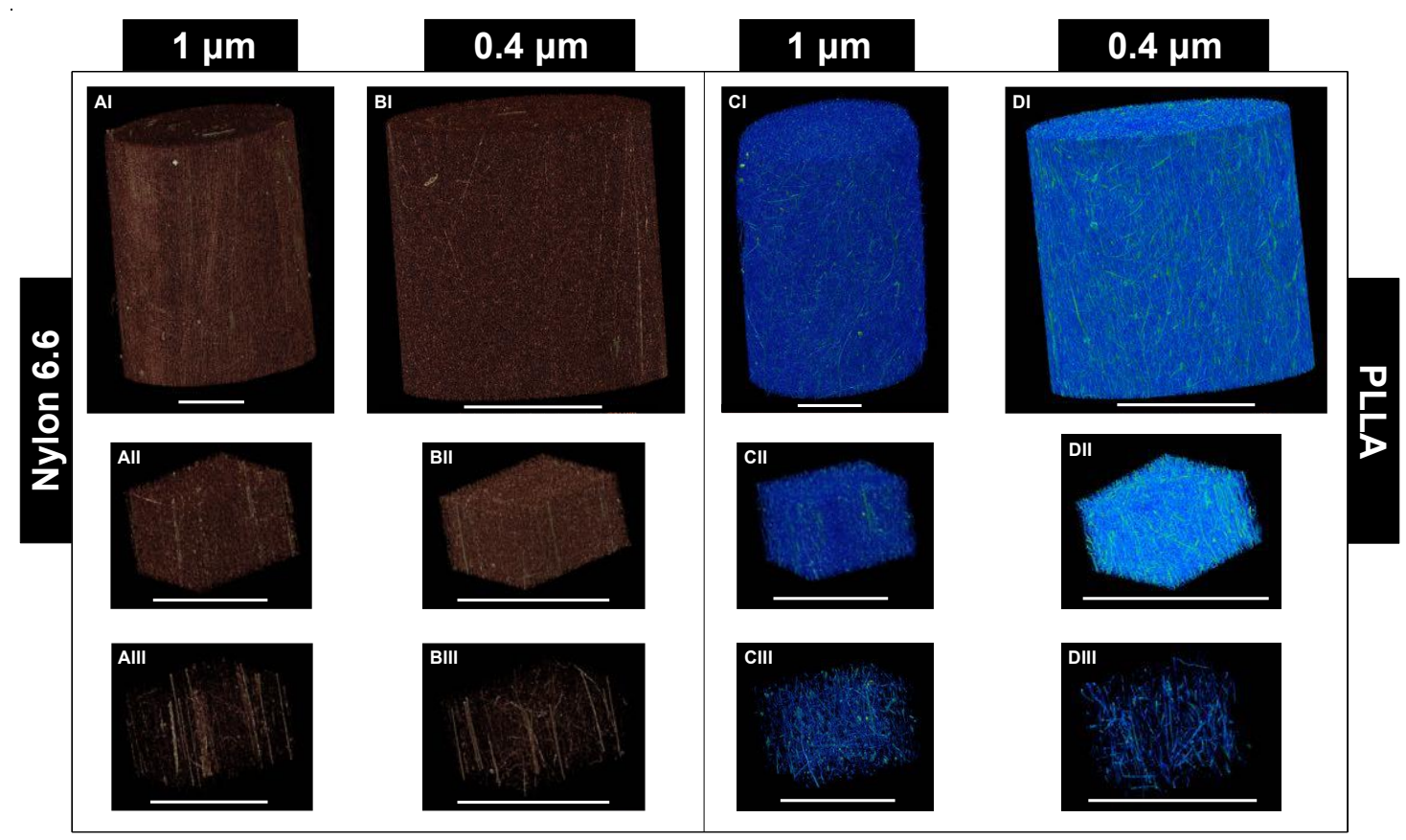

Fig. 4. XCT images of (A, B) the Nylon 6.6 and (C, D) PLLA bundles at (A, C) 1.0 micrometer voxel size, and (B, D) 0.4 micrometer voxel size (scale bars $=200$ micrometer). (I) Overview of the bundles for the two different voxel sizes showing all the nanofibers. (II) and (III) Crop of an internal volume of the bundles with different thresholding.

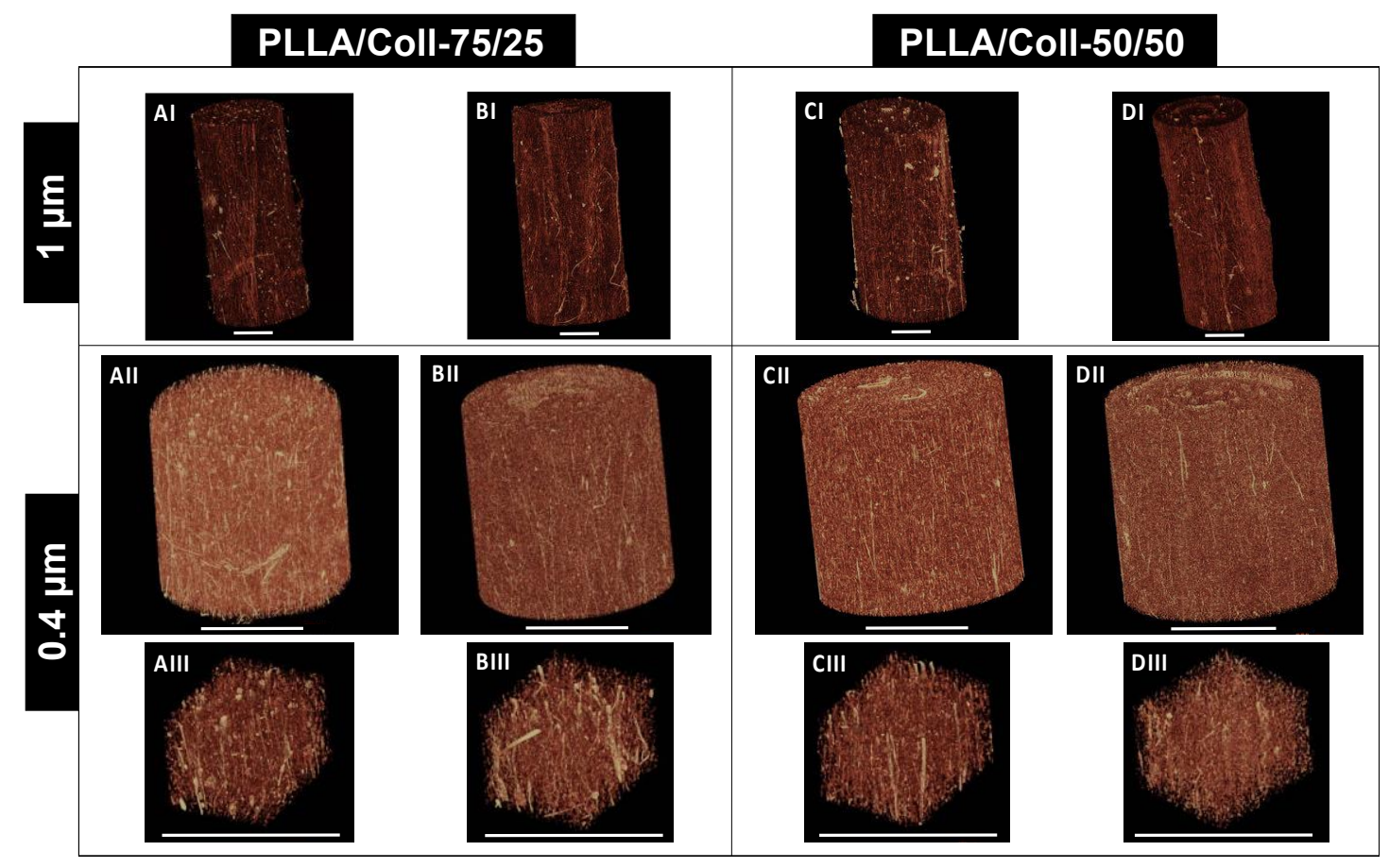


Fig. 5. XCT images of the PLLA/Coll-75/25 bundles (A) as-spun and (B) crosslinked, and of the PLLA/Coll-50/50 bundles (C) as-spun, and (D) crosslinked (scale bar $=200$ micrometer). Two voxel sizes are shown: (I) the 1.0 micrometer scan shows the nanofibers of the bundles for the different compositions; the 0.4 micrometer scan is shown as (II) an overview of the entire bundle, and (III) as a crop of an internal volume, showing the nanofibers.

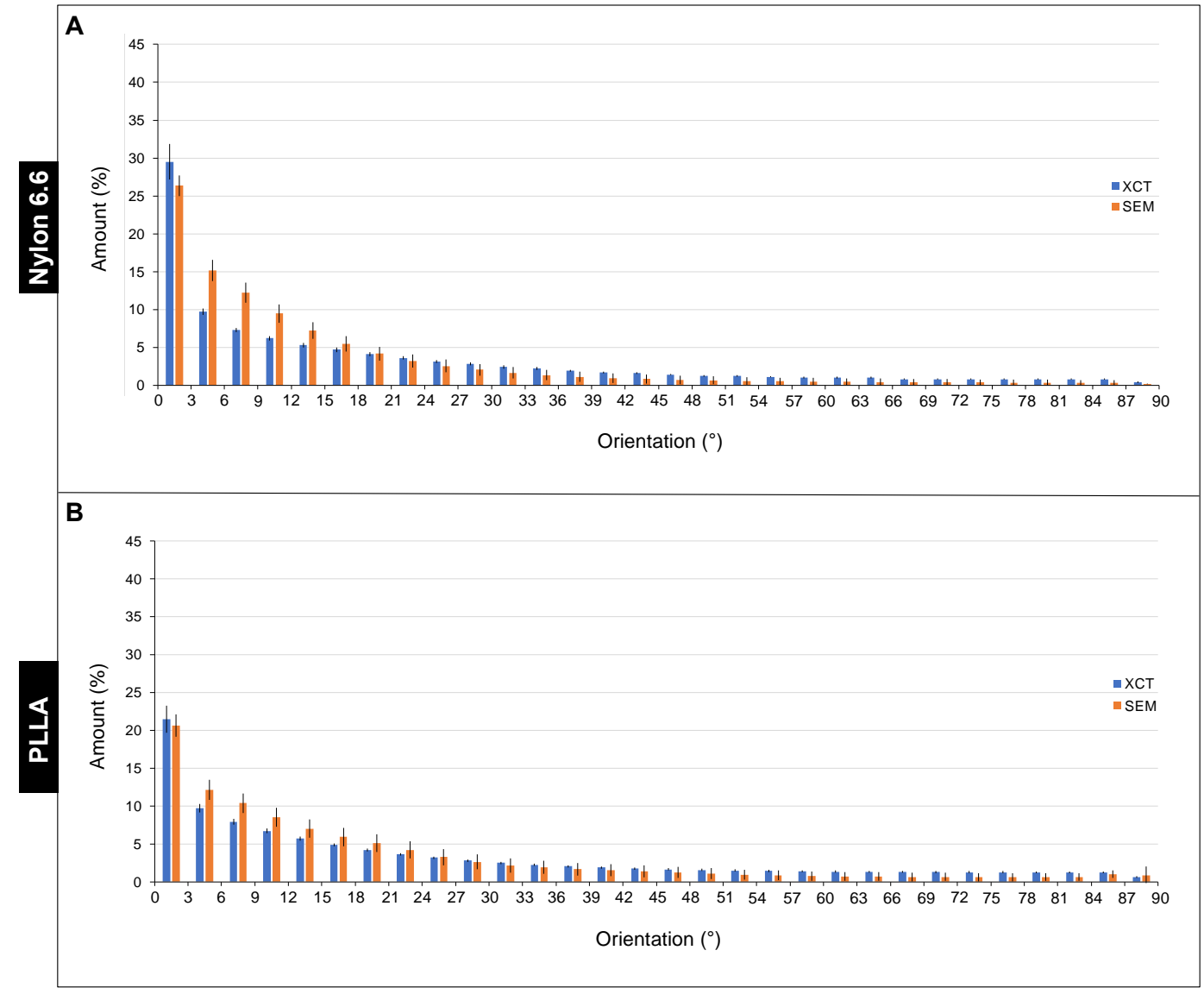

Fig. 6. Comparison between the Directionality measured on the XCT at 0.4 micrometer voxel size (blue bars) and SEM images at $8000 x$ magnification (orange bars) for two compositions: (A) Nylon 6.6 bundles and (B) pure PLLA bundles. An angle of $0^{\circ}$ means that the nanofibers were aligned with the axis of the bundles, an angle of $90^{\circ}$ means that the nanofibers were perpendicular to the bundle. Mean and standard deviation between images of the same specimen are plotted. 


\section{PLLA/Coll-75/25}

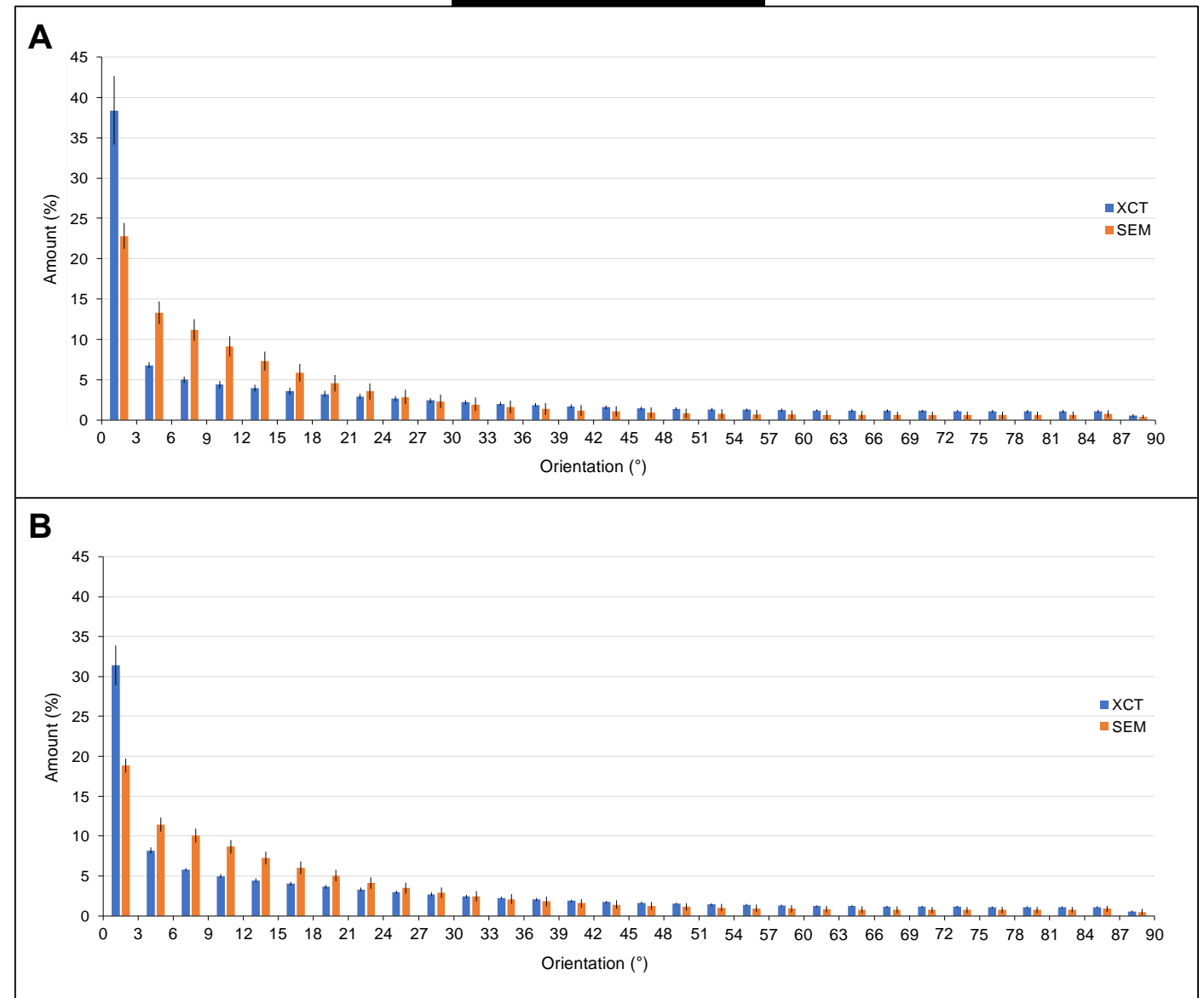

Fig. 7. Comparison between the Directionality measured on the XCT at 0.4 micrometer voxel size (blue bars) and SEM images at 8000x magnification (orange bars) for the PLLA/Coll-75/25 bundles (A) as-spun bundles and (B) crosslinked. An angle of $0^{\circ}$ means that the nanofibers were aligned with the axis of the bundles, an angle of $90^{\circ}$ means that the nanofibers were perpendicular to the bundle. Mean and standard deviation between images of the same specimen are plotted. 


\section{PLLA/Coll-50/50}

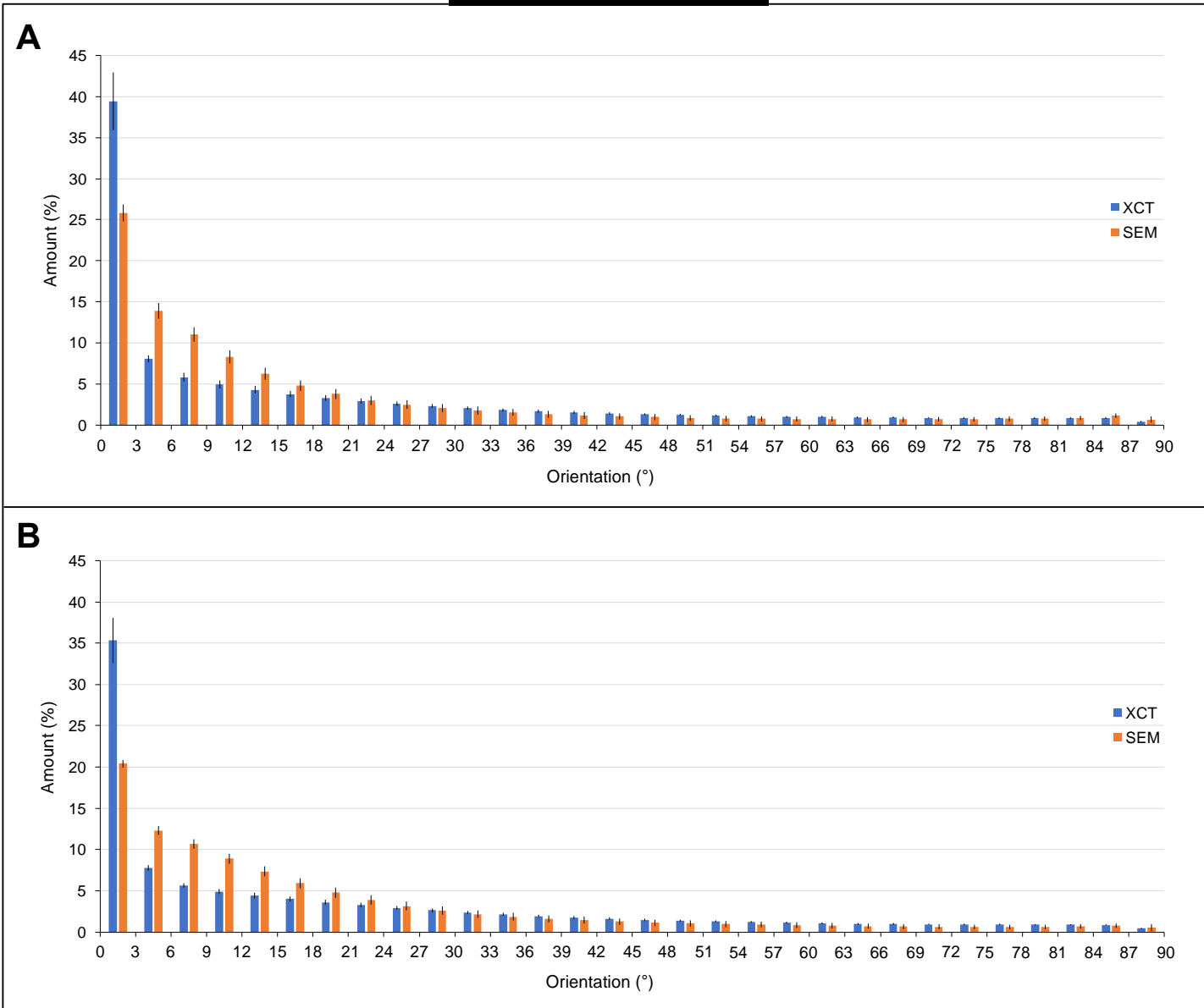

Fig. 8. Comparison between the Directionality measured on the XCT at 0.4 micrometer voxel size (blue bars) and SEM images at 8000x magnification (orange bars) for the PLLA/Coll-50/50 bundles (A) as-spun bundles and (B) crosslinked. An angle of $0^{\circ}$ means that the nanofibers were aligned with the axis of the bundles, an angle of $90^{\circ}$ means that the nanofibers were perpendicular to the bundle. Mean and standard deviation between images of the same specimen are plotted. 